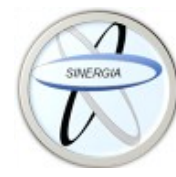

Revista Médica Sinergia

Vol. 6, Núm. 5, mayo 2021, e672

\title{
Delirio: una visión global
}

\section{Delirium: a global visión}

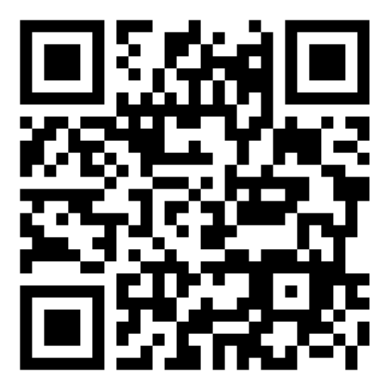

Recibido 18/03/2021
${ }^{1}$ Dra. Mariam Ramírez Solano Investigadora independiente, San José, Costa Rica

(i) https://orcid.org/0000-0003-2249-6767

${ }^{2}$ Dra. Alice Umaña Venegas Investigadora independiente, San José, Costa Rica

(i) https://orcid.org/0000-0002-4047-5006

Corregido 04/04/2021

Aceptado 05/04/2021

\section{RESUMEN}

El delirio es un estado de disfunción mental, de corta duración, habitualmente reversible que afecta la atención y las funciones cognitivas.

Puede desencadenarse a partir de múltiples condiciones en los diferentes servicios de asistencia sanitaria, desde la atención domiciliar, internamientos, post quirúrgicos y servicios de cuidados paliativos.

Existen estrategias de prevención y medidas tanto farmacológicas como no farmacológicas para el manejo del delirio que varían dependiendo del origen del mismo.

PALABRAS CLAVE: delirio; confusión; haloperidol; síndrome; desórdenes neurocognitivos; disfunción cognitiva.

\section{ABSTRACT}

Delirium is a status of mental disfunction, of short period of time, usually reversible, that affects the attention and cognitive functions. It can be triggered by multiple conditions in the different health care services, from home care, hospitalizations, postsurgical and palliative care services. There are prevention strategies and pharmacological and non-pharmacological measures for the management of delirium that vary depending on its origin.

KEYWORDS: delirium; confusion; haloperidol; syndrome; neurocognitive disorders; cognitive dysfunction.

${ }^{1}$ Médica general, graduada de la Universidad Latina de Costa Rica. Cód. MED14114. Correo: maraso09@hotmail.com

${ }^{2}$ Médica general, graduada de la Universidad Hispanoamericana. Cód. MED14272. Correo: alicevuv@gmail.com 


\section{INTRODUCCIÓN}

El término delirio se deriva del latín de- que significa fuera de y lira- que significa camino, lo que puede interpretarse como "fuera del camino" (1). Pese a que fue incluido por primera vez en la tercera edición del manual diagnóstico y estadístico de enfermedades mentales (DSM-3), ya desde la antigüedad se utilizaba el termino para hacer referencia a anomalías en el pensamiento y la conducta asociadas a fiebre por una patología orgánica $(2,3)$. También a lo largo de la historia se le ha conocido con otras denominaciones tal como síndrome confusional agudo, síndrome cerebral orgánico, psicosis postquirúrgica o encefalopatía tóxico-metabólica (2). Pese a que es una entidad patológica conocida desde antaño, no es infrecuente que este cuadro clínico no sea reconocido o manejado de forma óptima. Esta revisión tiene como objetivo brindar información actualizada sobre los criterios diagnósticos y el manejo de esta entidad nosológica, con el fin de brindar un abordaje adecuado, detección oportuna y manejo idóneo.

\section{MÉTODO}

Esta revisión se realizó mediante la búsqueda de investigaciones y revisiones actualizadas sobre el delirio o síndrome confusional agudo en diversas bases de datos con el apoyo de la Biblioteca Nacional en Salud y Seguridad Social (BINASSS).

Se excluyeron del estudio revisiones con más de 5 años de antigüedad y artículos de presentación de casos individuales.

Los estudios utilizados incluyen desde metaanálisis, artículos de revisión, guías de práctica clínica y estudios epidemiológicos.

\section{DEFINICIÓN}

El delirio es síndrome caracterizado por una disfunción cerebral de carácter agudo o subagudo, que no se explica por desórdenes neurocognitivos previos, y se presenta por múltiples etiologías orgánicas de forma reversible $(4,2)$. Su duración es variable al igual que el grado de afectación, clínicamente se caracteriza por alteración del nivel de conciencia y de la atención, además puede presentar adicionalmente afectación de funciones cognitivas y no intelectivas (2).

Dentro de las funciones cognitivas que se ven afectadas la memoria, la orientación, el leguaje y la percepción. Mientras que en el caso de las funciones no intelectivas existen alteraciones del estado afectivo, del ciclo de sueno vigilia, entre otras (5).

\section{EPIDEMIOLOGÍA}

Esta entidad afecta de un $15-30 \%$ de los pacientes durante la estancia hospitalaria, mientras que en la comunidad presenta una prevalencia de aproximadamente 1-2\% $(1,5)$.

Se presenta con mayor frecuencia en hombres, principalmente en adultos mayores, e incluso según estudios por cada año de vida posterior a los 50 años el riesgo de delirio aumenta en un 10\% (6).

El síndrome confusional agudo, es la complicación post quirúrgica más frecuente en el adulto mayor, con una frecuencia del $15-20 \%$. Si se trata de una cirugía de riesgo tal como una cirugía por fractura de cadera o cirugía cardiaca, dicha frecuencia aumenta hasta en un $50 \%$ de los casos (2).

En las unidades de cuidados intensivos, los pacientes que se encuentran con ventilación mecánica presentan una incidencia acumulada que supera el $75 \%$ al combinarse con estupor o coma. Mientras que, en el departamento de emergencias, el delirio se presenta en el $10-15 \%$ de los adultos mayores que se encuentran ingresados en este (7).

En la población pediatría se reporta una prevalencia muy variable que va desde 12 a $65 \%$ en unidades de cuidados intensivos 
médicas y quirúrgicas, presentándose una prevalencia más alta en niños menores de 5 años, con trastornos del neurodesarrollo o enfermedades graves (8).

\section{FACTORES DE RIESGO}

En cuanto a los factores de riesgo, estos de clasifican en factores predisponentes $y$ factores desencadenantes, en la tabla 1 se describen de forma detallada (7). Los factores predisponentes son todos los que aumentan la vulnerabilidad del paciente a desarrollar delirio, mientras que los factores desencadenantes son todas las entidades patológicas, procedimientos o drogas que pueden causar síndrome confusional agudo. En cuanto a los factores predisponentes se encuentra la edad avanzada especialmente en adultos mayores de 80 años, la cual se ha observado que aumenta el desarrollo de delirio asociado a una incidencia incrementada de demencia (9). Además, el sexo masculino, historia de episodios previos de delirio, baja escolaridad, antecedentes de evento cerebrovascular, demencia, deterioro funcional y fragilidad.

Dentro de los factores precipitantes figuran los cateterismos vesicales, las vías centrales o periféricas, ingresos a unidades de cuidados intensivos o unidades coronarias, patologías neurológicas agudas, trastornos hidroelectrolíticos, traumas, infecciones, cirugías y ciertos fármacos (1).

Sobre el riesgo postoperatorio de delirio, este varía dependiendo del procedimiento quirúrgico realizado, en el caso de las cirugías por otorrinolaringología y cirugía general el riesgo es de un 12 y $13 \%$ respectivamente, cirugía abdominal mayor presenta un riesgo mayor al $50 \%$, mientras que en casos de cirugías cardiacas existe un riesgo por encima del $51 \%$ (4).

Los fármacos que con mayor frecuencia precipitan el desarrollo de delirio son los benzodiacepinas, antihistamínicos,

anticolinérgicos, anticonvulsivantes, analgésicos y la deprivación de drogas, fármacos o alcohol. El delirio por abstinencia representa una subcategoría de delirio que se presenta tras la discontinuación abrupta de una droga o fármaco de uso prolongado y clínicamente se manifiesta con agitación psicomotriz, alucinaciones e ideas delirantes (10).

\section{FISIOPATOLOGÍA}

El mecanismo exacto por el cual se desarrolla el delirio no está claramente identificado, sin embargo, es probable que las manifestaciones del delirio tengan origen en la alteración de múltiples neurotransmisores, factores proinflamatorios y determinación genética de la respuesta ante factores predisponentes del delirio (11).

Existe una desregulación en el equilibrio de producción y función de neurotransmisores que genera una disfunción en la actividad neuronal aunado a la liberación de sustancias proinflamatorias que provocan alteración de la perfusión cerebral, disfunción endotelial y alteración en la permeabilidad de la barrera hematoencefálica (4).

En cuanto a las anomalías en el sistema de neurotransmisores, el principal es el déficit colinérgico, en donde la deficiencia de acetilcolina explica el desarrollo de delirio desencadenado por fármacos anticolinérgicos en pacientes con factores predisponentes.

Además, existen patologías tales como la demencia tipo Alzheimer, demencia con cuerpos de Lewy o demencia por Parkinson que presentan un déficit colinérgico crónico a raíz de la neurodegeneración en el núcleo basal de Meynert, el cual es el principal productor de acetilcolina en el sistema nervioso central.

Otro neurotransmisor involucrado es la dopamina, ya que los niveles elevados de esta pueden desencadenar delirio, razón por 


\begin{tabular}{|c|c|}
\hline Factores predisponentes & Factores precipitantes \\
\hline $\begin{array}{l}\text { - } \text { Edad mayor a } 65 \text { años Sexo masculino } \\
\text { - } \text { Deterioro cognitivo } \\
\text { - Historia previa de delirio } \\
\text { - Pluripatología } \\
\text { - Patologías neurológicas } \\
\text { - Deterioro de funcionalidad } \\
\text { - Deprivaciones sensoriales (déficit visual, } \\
\text { - déficit auditivo) } \\
\text { - Deficiencias nutricionales } \\
\text { - } \text { Abuso de sustancias } \\
\text { - Trastornos psiquiátricos (depresión, ansiedad) }\end{array}$ & $\begin{array}{l}\text { - } \text { Trastornos electrolíticos } \\
\text { - } \text { Abstinencia de sustancias o fármacos } \\
\text { - } \text { anticonvulsivantes, antagonistas H2, AINES, } \\
\text { - } \text {-dopa). } \\
\text { - } \text { Polifarmacia } \\
\text { - } \text { Quertermia - hipotermia } \\
\text { - Traumas } \\
\text { - } \text { Procedimientos quirúrgicos } \\
\text { - } \quad \text { Trastornos del sueño } \\
\text { - } \text { Dolor } \\
\text { - } \text { Fiebre } \\
\text { - Hipoxia } \\
\text { - Infecciones } \\
\text { - Ingresos hospitalarios }\end{array}$ \\
\hline
\end{tabular}

Fuente. Acute Brain Failure Pathophysiology, Diagnosis, Management, and Sequelae of delirium, 2017.

la cual la L-dopa puede desencadenar delirio.

Dentro de los demás neurotransmisores estudiados en la patogenia del delirio, se han documentado niveles elevados de norepinefrina y glutamato, además de niveles bajos de melatonina la cual se encuentra estrechamente ligada con los ciclos de sueño - vigilia $(4,11)$.

\section{DIAGNÓSTICO}

El delirio es inespecífico, frecuentemente es una manifestación reversible de enfermedad aguda que puede tener diversas etiologías, que incluye recuperación adecuada de un estado exagerado si se trata su causa (12). La sospecha clínica es la herramienta más importante.

La alteración de la atención, conciencia, percepción, el pensamiento, el ciclo sueño vigilia, así como su aparición aguda y la fluctuación de los síntomas son factores claves para el diagnóstico (9).
Los criterios de la Clasificación Internacional de las Enfermedades y Trastornos relacionados con la salud (CIE-10) como los de la DSM-5 siguen siendo el estándar de oro para el diagnóstico del delirio $(14,15)$. El diagnóstico se establece con los datos recolectados en la historia clínica, sin embargo, se puede complementar con algunas herramientas o escalas. El uso de escalas para evaluar la gravedad ha demostrado tener un valor predictivo en relación con la duración del internamiento, riesgo de institucionalización y mortalidad. Entre las escalas más utilizadas se encuentra Delirium Rating Scale - Revised 98 (DRS-R-98), la Memorial Delirium Assessment Scale o la CAM -s everity scale (CAM-S) (9).

El abordaje del paciente debe incluir anamnesis completa, valoración del estado neurológico y examen físico exhaustivo. Uso de estudios complementarios como hemograma, leucograma, electrolitos, prueba funciona renal y hepática, examen general de orina, hemocultivo, punción 
lumbar indicado si existe cefalea, fiebre o síntomas sugestivos de infección del sistema nervioso central, electroencefalograma en paciente con antecedente de trauma de cráneo, ictus o epilepsia. Tomografía axial computarizada del sistema nervioso central (SNC) cuando exista hallazgos de focalización neurológica, radiografía de tórax, electrocardiograma, entre otros. Indagar además sobre consumo de drogas o síndrome de abstinencia de alcohol.

La investigación adicional para las diversas etiologías depende de la clínica que presente el paciente, así como de la experiencia del clínico.

La causa del delirio puede ser multifactorial por lo que hay que tomar en consideración los diversos desórdenes neurocognitivos previos, así como descartar otro tipo de etiología médica. Entre los factores precipitantes y predisponente están el patrón de uso de medicamentos como opioides, benzodiacepinas, medicamentos anti nauseosos o antimigrañosos, estabilizadores del estado de ánimo que tiene actividad serotoninérgica. La inmunosupresión aumenta el riesgo de infecciones oportunistas en el SNC, metástasis a SNC, corrección rápida de trastornos metabólicos como en la hiponatremia, accidente cerebro vascular. Otras etiologías que provocan delirio son retención urinaria, impactación fecal, trastorno pulmonar y del miocardio, sepsis de tejido blando. Además, debemos tomar en cuenta la demencia, psicosis, depresión, paranoia como diagnósticos diferenciales. (16).

\section{TRATAMIENTO}

El objetivo del tratamiento debe de buscar limitar la morbilidad y mortalidad incluido el riesgo de deterioro cognitivo a largo plazo del paciente. Utiliza múltiples estrategias preventivas como educación continua del personal de salud y de los cuidadores, búsqueda de las causas médicas del delirio, optimización del manejo de dolor evitando el uso de opioides y de medicamentos que tiene alto riesgo de provocar delirio (por ejemplo, anticolinérgicos, anti dopaminérgicos, sedantes, antipsicóticos).

Podemos dividirlo en tratamiento no farmacológico $y$ en tratamiento farmacológico.

\section{Tratamiento no farmacológico}

Las intervenciones no farmacológicas son parte fundamental del tratamiento en el delirio. Entre las intervenciones que se sugieren se encuentra disminución en la perturbación del medio ambiente, permitir un área oscura para conciliar el sueño, así como durante el día haya adecuada iluminación, que se pueda optimizar la visión y audición en el medio donde se desenvuelva el paciente ya sea en su lugar de residencia o internamiento hospitalario, facilitar el uso de herramientas para orientar al paciente como calendario, relojes, etc.; evitar las restricciones físicas, proporcionar una adecuada ingesta de líquidos y nutrición (18).

La prevención es la forma más efectiva para evitar que se desarrolle el delirio. Los enfoques no farmacológicos redujeron significativamente la incidencia de delirio y recaída. Según una revisión de Cochrane sobre la prevención del delirio encontró una evidencia de moderada calidad donde indica que las intervenciones no farmacológicas son efectivas para la prevención del delirio, pero de menor impacto para disminuir la gravedad o duración del delirio (12).

\section{$\underline{\text { Tratamiento farmacológico }}$}

El manejo principal del delirio está basado en el tratamiento no farmacológico debido a que no existen medicamentos aprobados por la FDA. 
No hay impacto importante en la duración o severidad. La mayoría de los ensayos farmacológicos publicados no informan diferencias en las tasas o la duración del delirio (13). No hay una evidencia clara sobre el uso de medicamentos en el delirio (9).

Las guías también recomendaron no tratar el delirio hipoactivo farmacológicamente y no usar benzodiacepinas, excepto en el delirio relacionado con la abstinencia de alcohol o benzodiazepinas.

El uso de antipsicóticos no está asociado a la mejoría de la duración, gravedad, estancia hospitalaria o mortalidad, se recomienda el uso en situaciones de agitación extrema que represente un riesgo en la seguridad del paciente

Al igual que en los adultos a nivel pediátrico el tratamiento de delirio debe de seguir una evaluación sistemática desde la valoración de enfermedades subyacentes (infección, hipoxemia, manejo del dolor, trastorno electrolítico), factores iatrogénicos (mínima sedación, reconocimiento y tratamiento de abstinencia, evitar restricciones, revisión de uso de medicamentos), así como modificaciones ambientales (por ejemplo, movilización temprana, higiene del sueño, etc.) hasta el tratamiento farmacológico como antipsicóticos atípicos (17).

Los factores modificables como los medicamentos crónicos, el tratamiento de las infecciones, manejo de los factores ambientales son la piedra angular del manejo en el delirio $(19,20)$.

\section{CONCLUSIONES}

El delirio es un estado de disfunción mental, de corta duración, habitualmente reversible que afecta la atención y las funciones cognitivas.

Se asocia un incremento en el costo financiero en la atención médica, en la mortalidad y morbilidad a pesar de ser reconocido como un trastorno de duración limitada.
Una valoración integral incluiría la evaluación del estado neurológico basal previa al ingreso, así como la identificación de etiologías médicas subyacentes que podrían contribuir a cambios en el estado mental por ejemplo infecciones, trastorno hidroelectrolítico, hiperglucemia, entre otros. El tratamiento incluiría intervenciones no farmacológicas, identificación de posibles contribuyentes al delirio relacionados con la dosis o incorporación de medicamentos de uso reciente.

El tratamiento fundamental es la identificación y manejo de enfermedades médicas subyacentes.

El diagnóstico acertado abarca desde una buena historia clínica, un examen físico exhaustivo, así como el uso de herramientas de evaluación confiables que pueda orientar el diagnóstico.

No hay clara evidencia de uso de fármacos específicos en el deliro por lo que en algunos estudios se recomienda evitar medicamentos sedantes y antipsicóticos. En caso de utilizar medicamentos se recomienda iniciar con la dosis mínima y con precaución.

\section{Los autores declaran no tener conflicto de interés.}

\section{REFERENCIAS}

1. Pérez $Y$, Turro L, Mesa R, Turro E. Peculiaridades del síndrome confusional agudo en el anciano. MEDISAN. 2018; 22(5):543-550.

2. Álvarez MA, Pereira $V$, Llavero $M$, Ortuño $F$. Síndrome confusional.Med. 2018;12(69):40554061.

3. González F. Historia del delirium [Internet].researchgate.net:Editorial Universidad de Caldas ;1 ene 2009[consultado 2 ago 2020]. Disponible

en: https://www.researchgate.net/publication/304817 154_Historia_del_Delirium

4. Rengel K., Pandharipande P. Postoperative delirium. Presse Med. [Internet] 2018 [consultado 1 ago 2020];47(4): 53-64. Disponible en: https://www.sciencedirect.com/science/article/abs /pii/S075549821830157X?via\%3Dihub 
5. Parra S., Barragán D., Tejeiro J. Protocolo diagnóstico y terapéutico del síndrome confusional agudo. Med. 2019;12(74):4371-4375.

6. Maldonado JR. Acute Brain Failure Pathophysiology, Diagnosis, Management, and Sequelae of Delirium. Crit Care Clin 33 (2017) 461-519.

7. Marcantonio E.Delirium in Hospitalized Older Adults. N engl j med. 2017;377:1456-1466.

8. Patel K., Bell MJ. Delirium in Pediatric Critical Care.Pediatr Clin N Am 64 (2017) 1117-1132.

9. Setters B., Laurence M. Delirium. Prim Care Clin Office Pract 44 (2017) 541-559. 559 http://dx.doi.org/10.1016/j.pop.2017.04.010.

10. Torrales J., Barrios I. El delirium: una actualización para Internistas y Psiquiatras. Rev. virtual Soc. Parag. Med. Int. setiembre 2017; 4 (2):54-64.

11. Kasper D., Hauser S., Larry J., Fauci A., Longo D., Loscalzo J. Harrison Principios de Medicina Interna. Edición 19. México D.F. Mc Graw-Hill. 2015.

12. David J. Dries, MSE, MD. Critical Care Update. Delirium. Air Medical Journal 37 (2018) 225-228. Elsevier.

13. Siddiqi N, Harrison J, Clegg A, et al. Interventions for preventing delirium in hospitalised non-ICU patients. Cochrane Database Syst Rev 2016;(3):CD005563.
14. Organización Mundial de la Salud. Clasificación Internacional de Enfermedades CIE-10. SId.Cu. 1992.

15. Association AP. DSM 5. American Journal of Psychiatry. 2013.

16. María de Lourdes Ramírez Echeverría; Manju Paul. Delirium. StatPearls. Treasure Island (FL): StatPearls Publishing; 2020 Jan-. NCBI Bookshelf.

17. Anita K. Patel, MDa, Michael J. Bell, MDb, Chani Traube, MD. Delirium in Pediatric Critical Care. Pediatr Clin N Am 64 (2017) 1117-1132. http://dx.doi.org/10.1016/i.pcl.2017.06.009

18. M.A. Álvarez-Mona, V. Pereira-Sáncheza , M. Llavero-Valerob y $F$. Ortuñoa. Síndrome confusional. Medicine. 2018;12(69):4055-6.

19. Trogrlić $Z$, van der Jagt $M$, Lingsma $H$, Gommers $D$, Ponssen $\mathrm{HH}$, Schoonderbeek JFJ, Schreiner F, Verbrugge SJ, Duran S, Bakker J, Ista E. Improved Guideline Adherence and Reduced Brain Dysfunction After a Multicenter Multifaceted Implementation of ICU Delirium Guidelines in 3,930 Patients. Crit. Care Med. 2019 Mar;47(3):419-427. [PubMed: 30608279].

20. 20.Sweeney J. Impacting Delirium in the Trauma ICU Utilizing the ICU Liberation Collaborative Benchmark Report. J Trauma Nurs. 2018 Nov/Dec;25(6):348-355. [PubMed: 30395033]. 